\title{
Formation and stabilization of multiple ball-like flames at earth gravity
}

\author{
Zhen Zhou ${ }^{\mathrm{a}, *}$, Yuriy Shoshin ${ }^{\mathrm{a}}$, Francisco E. Hernández-Pérez ${ }^{\mathrm{b}}$, Jeroen A. van Oijen ${ }^{\mathrm{a}}$, Laurentius \\ P.H. de Goey ${ }^{\mathrm{a}}$ \\ ${ }^{a}$ Department of Mechanical Engineering, Eindhoven University of Technology, P.O. Box 513, 5600MB Eindhoven, \\ The Netherlands \\ ${ }^{b}$ Clean Combustion Research Center, King Abdullah University of Science and Technology, Thuwal 23955-6900, \\ Saudi Arabia
}

\begin{abstract}
Near-limit low-Lewis-number premixed flame behavior is studied experimentally and numerically for flames of $\mathrm{H}_{2}-\mathrm{CH}_{4}$-air mixtures that are located in a $55 \mathrm{~mm}$ diameter tube and below a perforated plate in a downward mixture flow. A combustion regime diagram is experimentally identified in terms of equivalence ratio and ratio of $\mathrm{H}_{2}$ to $\mathrm{CH}_{4}$ (variation of fuel Lewis number). Planar flames, cell-like flames, distorted cap-like flames, and arrays of ball-like flames are progressively observed in the experiments as the equivalence ratio is decreased. The experimentally observed ball-like lean limit flames experience chaotic motion, which is accompanied by sporadic events of flame splitting and extinction, while the total number of simultaneously burning flamelets remains approximately the same. In separate experiments, the multiple ball-like lean limit flames are stabilized by creating a slightly non-uniform mixture flow field. The $\mathrm{CH}^{*}$ chemiluminescence distributions of the lean limit flames are recorded, showing that the ball-like lean limit flame front becomes more uniform in intensity and its shape approaches a spherical one with the increase of $\mathrm{H}_{2}$ content in the fuel. Numerical simulations are performed for single representative flames of the array of stabilized flamelets observed in the experiments. The simulated ball-like lean limit flame is further contrasted with the single ball-like flame that forms in a narrow tube $(13.5 \mathrm{~mm}$ inner diameter) with an iso-thermal wall. The numerical results show that the ball-like lean limit flames present in the array of ball-like flames are more affected by the buoyancy-induced recirculation zone, compared with that in the narrow tube, revealing why the shape of the ball-like flame in the array deviates more from a spherical one. All in all, the wall confinement is not crucial for the
\end{abstract}


formation of ball-like flames at terrestrial gravity.

Keywords: Low Lewis Number, Lean Flammability Limit, Ball-like Flame, Hydrogen-Methane

\section{Introduction}

Hydrogen addition to hydrocarbon fuels is of great interest from a practical point of view due to its potential utilization in burners operating at ultra lean conditions, which can reduce pollutant emissions and increase flame stability. Therefore, much attention is given to the combustion characteristics of hydrogen-containing premixed flames at ultra-lean conditions. Despite the fact that the understanding of hydrogen combustion has been greatly improved in recent years [1], the understanding of the near-limit flame behavior of hydrogen-containing mixtures is still incomplete because of the rich and complex phenomena displayed by such low Lewis number flames.

For low Lewis number flames, a unique flame structure exists near the flammability limit at micro-gravity, that is the so-called flame ball. Zeldovich [2] first theoretically predicted a purely diffusion controlled flame with a steady radius burning in an unconfined medium of premixed reactants. While the solution found by Zeldovich at adiabatic conditions is unstable, he further hypothesized that heat loss might stabilize the flame balls. Ronney [3] unexpectedly observed spherical flamelets (flame balls) in a drop tower experiment. In subsequent parabolic flight experiments, Ronney et al. [4] confirmed that spherical flamelets can exist in different near-limit low-Lewis-number mixtures. However, these spherical flamelets were strongly affected by gravity fluctuations. Abid et al. [5] experimentally observed stable flame balls in a 10-second drop tower experiment. Later, space shuttle experiments [6] showed that flame balls not only can burn for a long time but also drift away from each other.

Kagan and Sivashinsky [7] numerically found that a two-dimensional (2D) flame front breaks into separate closed-front flamelets at zero-gravity conditions in the presence of sufficiently high radiative heat loss. The closed-front flamelets were further theoretically studied by Brailovsky and Sivashinsky [8], showing that, besides the stationary flame ball, there may also exist so-called travelling flame balls. Fursenko and Minaev [9] numerically simulated the three-dimensional flame

\footnotetext{
${ }^{*}$ Corresponding author.

Email address: Z.Zhou1@tue.nl (Zhen Zhou)
}

Preprint submitted to Combustion and Flame 
propagation in a diverging channel at zero-gravity condition, observing multiple ball-like flames accompanied by splitting and extinguishing when the radiative heat loss is sufficiently large. Recently, Takase et al. [10] experimentally observed that a ball-like low-Lewis-number flame exists near the lean limit in a low-stretch counterflow burner at micro-gravity conditions. Moreover, Fursenko et al. [11] experimentally and numerically identified the combustion regimes of low-Lewis-number stretched premixed flames at micro-gravity conditions, and predicted a set of unstable ball-like flames near the lean limit. It should be noted that all the above studies were conducted at micro-gravity conditions or zero-gravity conditions.

Shoshin and de Goey [12] observed a transition from a cell-like propagating flame to a ball-like flame in hydrogen-methane-air mixtures near lean limit in experiments on flame propagation in a tube under normal gravity. Shoshin et al. [13] further studied this ball-like lean limit flame by experiments and numerical simulations. Subsequently, a ball-like lean limit flame was stabilized in a downward mixture flow [14]. A detailed comparison between these experimental and numerical ball-like flames has been conducted by Hernández-Pérez et al. [14]. However, it remains unclear how crucial the wall confinement is for the formation of ball-like flames. In order to understand the confinement effect, the following two questions have to be answered:

1. Can multiple ball-like flames appear in a space much larger than the size of the ball-like flames at normal gravity conditions? There is a reason to believe that there may exist a symmetric structure of multiple ball-like flames at normal gravity. Groups of unsteady and small flame structures have been observed earlier in experiments on the propagation of ultra-lean hydrogen-containing flames in a wide $(50 \mathrm{~mm})$ tube at normal gravity conditions $[12,15,16,17]$. Those flamelets, however, were strongly affected by the convective flow, resulting in an irregular flame shape, and were described as not closed "flame caps" instead of ball-like flame structure. Still, multiple ball-like flames at normal gravity might be attainable by reducing convection.

2. If so, what are the differences between ball-like flames in a relative large space and ball-like flames confined in small tubes?

In this regard, the aim of present study is to elucidate the above questions. An experimental 
setup is developed to investigate combustion regimes of the low-Lewis-number flames in a relatively large space at normal gravity conditions in a downward mixture flow. Using a downward flow allows to localize buoyancy-affected flames within a short test region and to perform a more detailed study comparable to the case of propagating flamelets. The near-limit combustion regimes in a downward mixture flow at normal gravity condition are studied for $\mathrm{H}_{2}-\mathrm{CH}_{4}$-air mixtures at different $\mathrm{H}_{2} / \mathrm{CH}_{4}$ ratios and equivalence ratios $(\phi)$. Numerical simulations are performed in a $2 \mathrm{D}$ cylindrically symmetric domain at conditions approximately representing the ones for a single unit of an array of stabilized flamelets observed in the experiments, and further contrasted with a corresponding flame in a narrow tube. In the following sections, the experimental setup and numerical approach are presented, and the experimental and numerical results are discussed. Finally, concluding remarks are given.

\section{Experimental setup}

A schematic of the experimental setup is shown in Fig. 1(a). The burner consists of a cylindrical plenum chamber with a diameter of $100 \mathrm{~mm}$ and a height of $200 \mathrm{~mm}$ and a quartz tube with an inner diameter of $55 \mathrm{~mm}$ and a height of $40 \mathrm{~mm}$. The unburned mixture first flows into the plenum chamber, is preconditioned by the metal foam, and then flows into the quartz tube through a cylindrically perforated plate with a diameter of $55 \mathrm{~mm}$, thickness of $2 \mathrm{~mm}$ and uniformly spaced with a $0.4 \mathrm{~mm}$ pitch and hexagonal pattern for holes (pore size: $0.3 \mathrm{~mm}$ ), as shown in Fig. 1(b). The metal foam with mean pore size of $1.0 \mathrm{~mm}$ is located at $80 \mathrm{~mm}$ from the top of the plenum chamber. Thereby, a uniform mixture flow at the top end of the quartz tube is produced. The composition of the unburned mixture is controlled by the mass flow controllers with an uncertainty of less than $1 \%$. In the experiments, the unburned mixture is ignited at the bottom of the burner at a sufficiently large equivalence ratio and then the equivalence ratio is reduced in a step-wise manner. Two AVT-PIKE F-032b CCD-cameras are employed to record the natural chemiluminescence of the flames from the side of the tube and from the bottom of the tube (with the help of a $45^{\circ}$ mirror). The entire tube wall is cooled by the cooling air and the maximum wall temperature is less than 
$320 \mathrm{~K}$. For the binary fuel-blend/air mixture, the equivalence ratio, $\phi$, is defined as follows:

$$
\phi=\frac{\left(F_{\mathrm{CH}_{4}}+F_{\mathrm{H}_{2}}\right) / O}{\left[\left(F_{\mathrm{CH}_{4}}+F_{\mathrm{H}_{2}}\right) / O\right]_{\mathrm{st}}}
$$

where $F_{i}$ is the mass of fuel, with $i=\mathrm{CH}_{4}$ and $\mathrm{H}_{2}, O$ is the mass of oxidizer, and the subscript, st, denotes the stoichiometric state.

In order to stabilize the multiple lean limit flames, in some experiments a corrugated wire mesh is installed at $10 \mathrm{~mm}$ below the perforated plate to introduce small random spatial variations of the inlet velocity. The wire mesh is gently punched by a steel ball of $4 \mathrm{~mm}$ diameter at random positions, producing $2 \mathrm{~mm}$ deep bumps on the mesh surface. The $\mathrm{CH}^{*}$ chemiluminescence for the lean limit flames that are stabilized below the middle part of perforated plate and not affected by the tube wall is recorded by a AVT-PIKE F-032b CCD-camera, mounted with an interference filter (430 $\mathrm{nm}$ and bandwidth $10 \mathrm{~nm}$ ). The chemiluminescence images show a line-of-sight integrated emission intensity of cylindrically symmetric flames. The in-plane radial emission intensity distribution is recovered by Abel inversion. The conditions of experiments are listed in Table 1. In order to compare the lean limit flames obtained in the present study with the previously studied lean limit flames in a tube with a much smaller inner diameter of $13.5 \mathrm{~mm}$ [14], the inlet velocities in the present experiments are set equal to the velocity near blow off limit for the lean limit flames in the tube with an inner diameter of $13.5 \mathrm{~mm}$. It should be mentioned that, with higher inlet velocity in the experiments, the lean limit flames are more affected by the convective flow, resulting in multiple flamelets with irregular shapes as observed in Ref. [12]. However, the value of lean flammability limit is not affected by the inlet velocity when the inlet velocity is larger than 6.5 $\mathrm{cm} / \mathrm{s}$. When the inlet velocity is larger than $6.5 \mathrm{~cm} / \mathrm{s}$, the heat loss to the burner deck has almost no influence on the lean flammability limit.

\section{Experimental observations and discussion}

Figure 2 shows the natural chemiluminescence distributions for $\mathrm{CH}_{4}$-air flames at three equivalence ratios. It is seen that, for a sufficiently large equivalence ratio, a planar flame forms. With the decrease of equivalence ratio, flat flame becomes wrinkled flame. As the lean combustion limit 


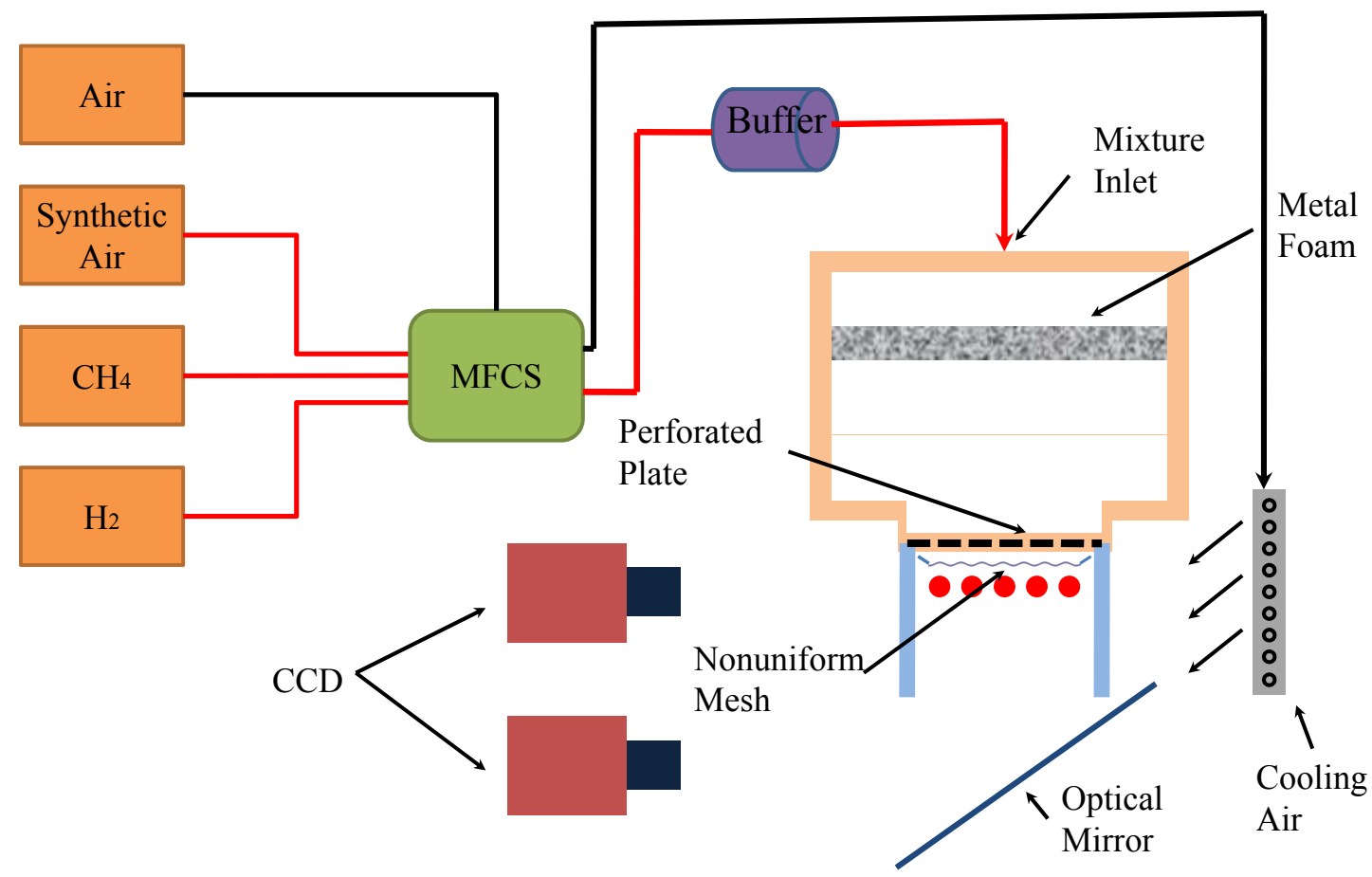

(a)

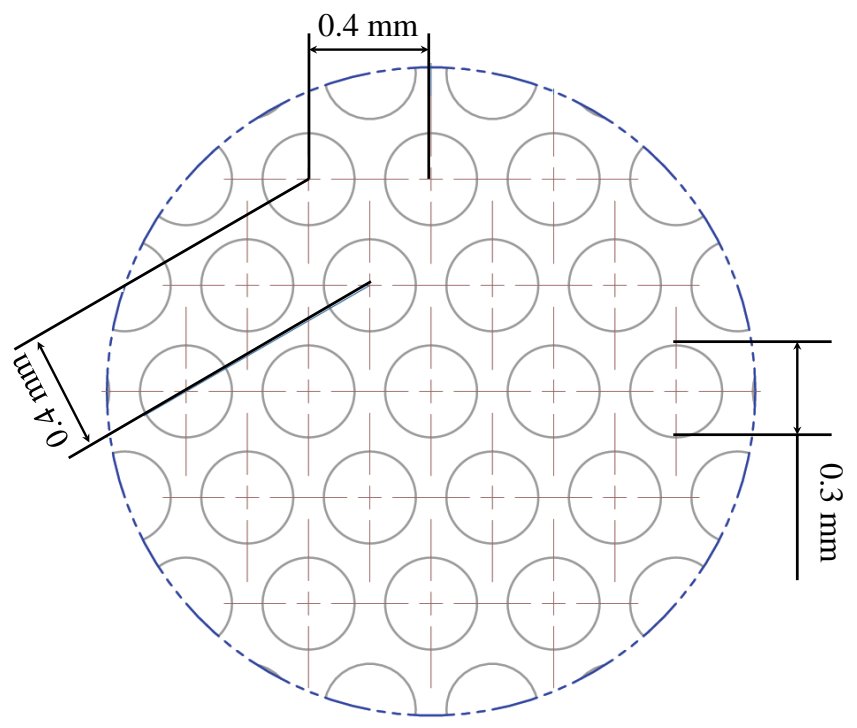

(b)

Figure 1: Schematic of experimental setup (a) and arrangement of holes in the perforated plate (b).

is approached, the flame front of the $\mathrm{CH}_{4}$-air flame becomes significantly wrinkled. Although the Lewis number of $\mathrm{CH}_{4}$ is close to unity, it lies below one and the burnt gas Markstein number of a $\mathrm{CH}_{4}$-air mixture near the lean flammability limit is negative [18]. Thus, preferential diffu- 
Table 1: Summary of conditions for the experiments with different ratio of $\mathrm{CH}_{4}$ and $\mathrm{H}_{2}$.

\begin{tabular}{ccc}
\hline Condition & Fuel Compositions & $V_{\text {in }}(\mathrm{cm} / \mathrm{s})$ \\
\hline I & $\mathrm{CH}_{4}$ & 0.58 \\
II & $20 \% \mathrm{H}_{2}+80 \% \mathrm{CH}_{4}$ & 0.72 \\
III & $40 \% \mathrm{H}_{2}+60 \% \mathrm{CH}_{4}$ & 0.72 \\
IV & $60 \% \mathrm{H}_{2}+40 \% \mathrm{CH}_{4}$ & 0.72 \\
V & $80 \% \mathrm{H}_{2}+20 \% \mathrm{CH}_{4}$ & 0.73 \\
\hline
\end{tabular}

sion effects can be expected. Furthermore, the Karlovitz number increases with the decrease of equivalence ratio due to the decrease of laminar burning velocity and the increase of the flame thickness, which enhances the preferential diffusion effects near the lean flammability limit. Notably, the flame front remains continuous without strong local extinction in flame cusps. However, with addition of $\mathrm{H}_{2}$ to $\mathrm{CH}_{4}$, the combustion behavior becomes more complex with the decrease of equivalence ratio owing to the enhancement of preferential diffusion effects, as compared with $\mathrm{CH}_{4}$-air flames. Figure 3 illustrates the evolution of the combustion regimes for $\left(80 \% \mathrm{H}_{2}+20 \%\right.$ $\mathrm{CH}_{4}$ )-air mixtures with decreasing equivalence ratio down to the lean flammability limit value. It is observed that, with decreasing equivalence ratio, the flat flame first changes to a set of small cell-like flames $(\mathrm{a}, \mathrm{b})$ which move chaotically. With a further reduction in equivalence ratio, the cell-like flames begin to merge together forming larger cell-like flames with irregular shape (c, d) (distorted cap-like flames). These cellular flame structures move chaotically and the size of these structures changes in time. With further decrease in equivalence ratio, the top part of the distorted cap-like flames becomes flatter and weaker, while the bottom part of the flame shows maximum luminosity (e, f). The cellular structures in Figs. 3(e, f) oscillate slightly and their size has almost no change in time. Finally, the distorted cap-like flames separate into a set of ball-like flames, which display chaotic motion $(\mathrm{g}, \mathrm{h})$.

The key features of the motion of the ball-like flames below the perforated plate are illustrated by a sequence of flame images in Fig. 4. It is found that some ball-like flames split into two secondary ball-like flames which are nearly identical to the primary one. Meanwhile, some balllike flames are blown down into the combustion products and extinguish. This evolution pattern of the ball-like flames is similar to that found by simulations in Ref. [9] for lean limit flames in a 


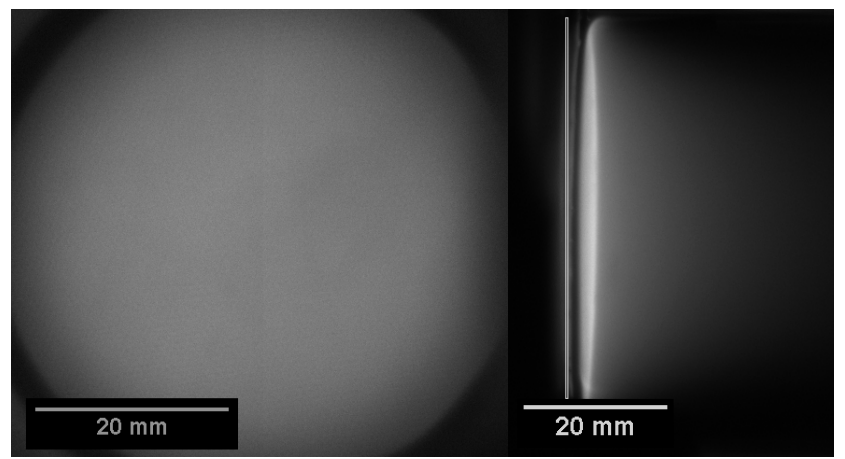

$\begin{array}{ll}\text { (a) } \phi=0.6 \text {, Bottom View } & \text { (b) } \phi=0.6 \text {, Side View }\end{array}$

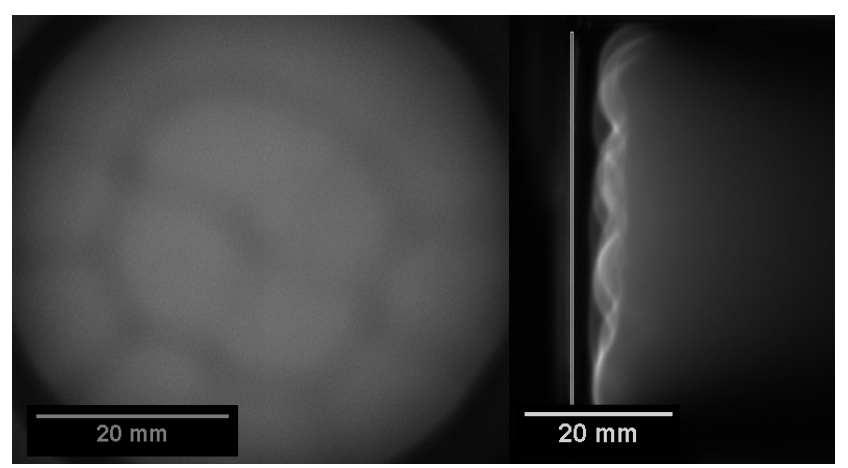

$\begin{array}{ll}\text { (c) } \phi=0.53 \text {, Bottom View } & \text { (d) } \phi=0.53 \text {, Side View }\end{array}$

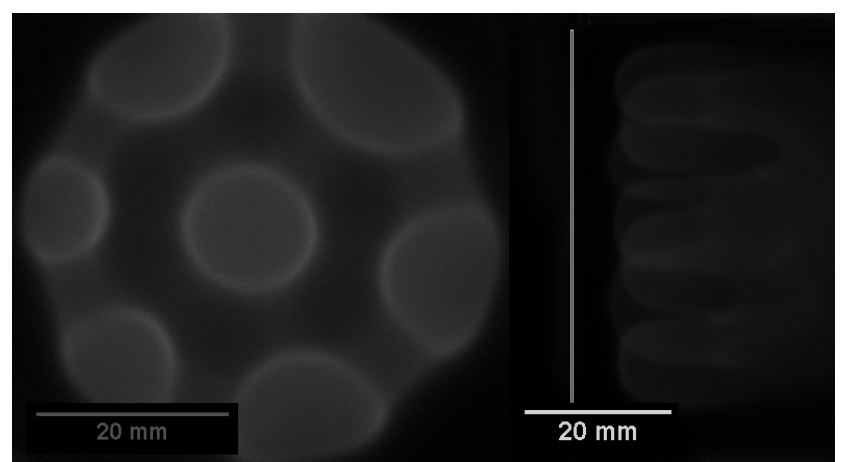

(e) $\phi=0.475$, Bottom View $\quad$ (f) $\phi=0.475$, Side View

Figure 2: Natural chemiluminescence distributions for $\mathrm{CH}_{4}$-air flames with different equivalence ratios. The light gray line indicates the surface of the perforated plate. Minimum stand-off distance: (b) $3.1 \mathrm{~mm}$; (d) $4.4 \mathrm{~mm}$; (f) 7.2 $\mathrm{mm}$.

diverging channel at zero-gravity conditions.

The combustion regimes that were encountered for other low-Lewis-number flames with different levels of $\mathrm{H}_{2}$ addition fall into the above described sequence of the combustion regimes. The observed regimes are mapped in Fig. 5 as function of the $\mathrm{H}_{2} / \mathrm{CH}_{4}$ ratio and equivalence ratio. The dotted line and dashed line in Fig. 5 just roughly separate the flat flames, cell-like flames and 


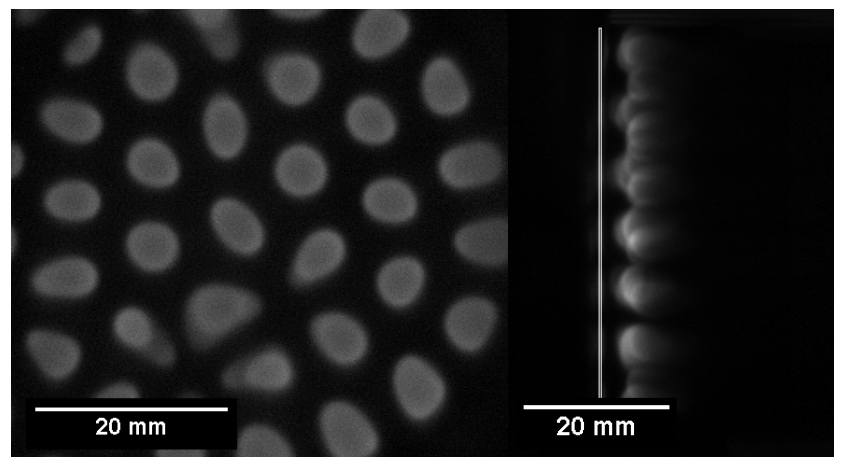

(a) $\phi=0.28$, Bottom View

(b) $\phi=0.28$, Side View

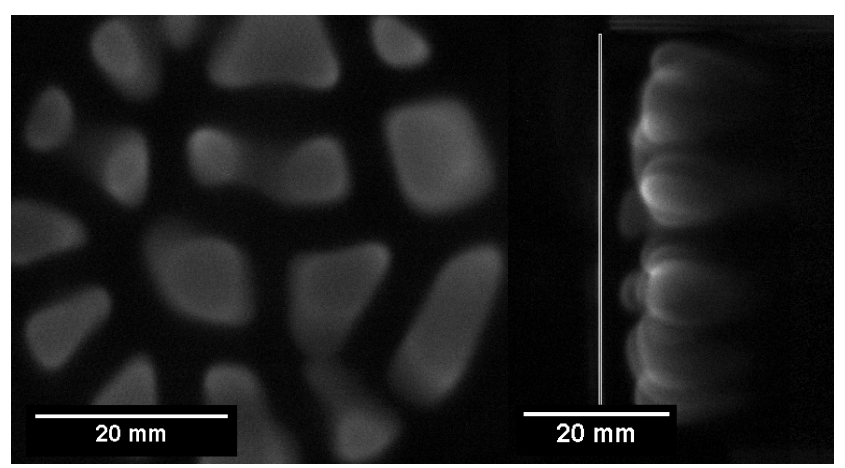

(c) $\phi=0.25$, Bottom View

(d) $\phi=0.25$, Side View

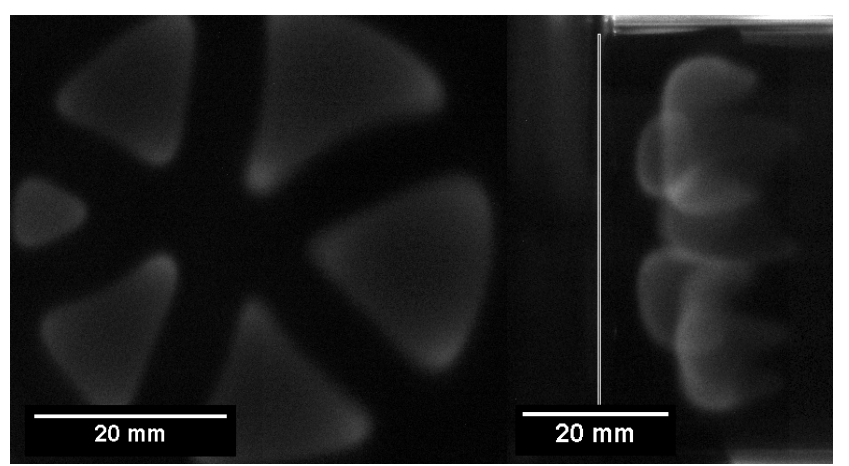

(e) $\phi=0.20$, Bottom View

(f) $\phi=0.20$, Side View

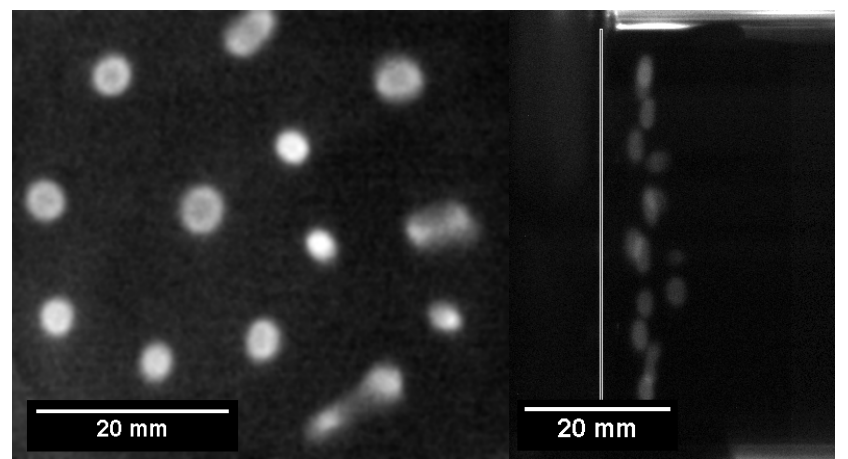

(g) $\phi=0.16$, Bottom View

(h) $\phi=0.16$, Side View

Figure 3: Natural chemiluminescence distributions for $\left(80 \% \mathrm{H}_{2}+20 \% \mathrm{CH}_{4}\right)$-air flames with different equivalence ratios. The light gray line indicates the surface of the perforated plate. Minimum stand-off distance: (b) $3.6 \mathrm{~mm}$; (d) $6.0 \mathrm{~mm}$; (f) $7.7 \mathrm{~mm}$; (h) $5.3 \mathrm{~mm}$. 


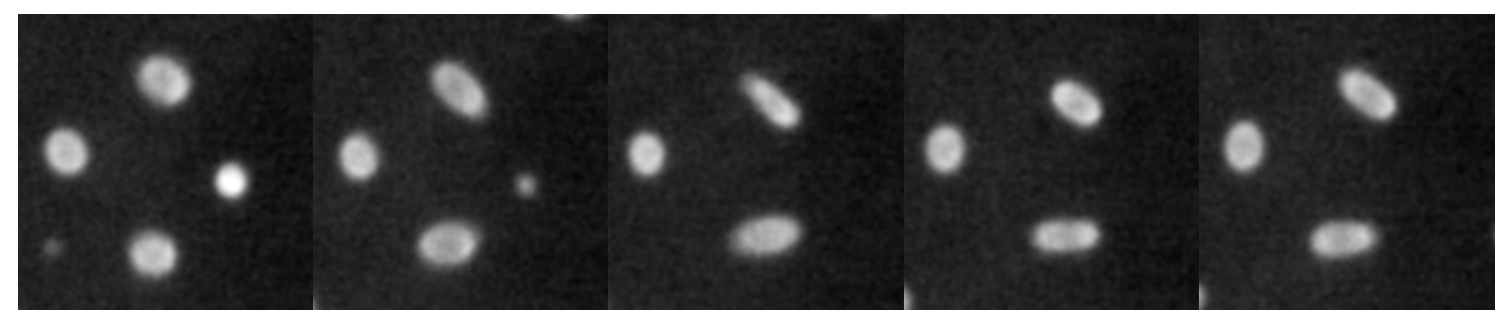

(a) 1

(b) 2

(c) 3

(d) 4

(e) 5

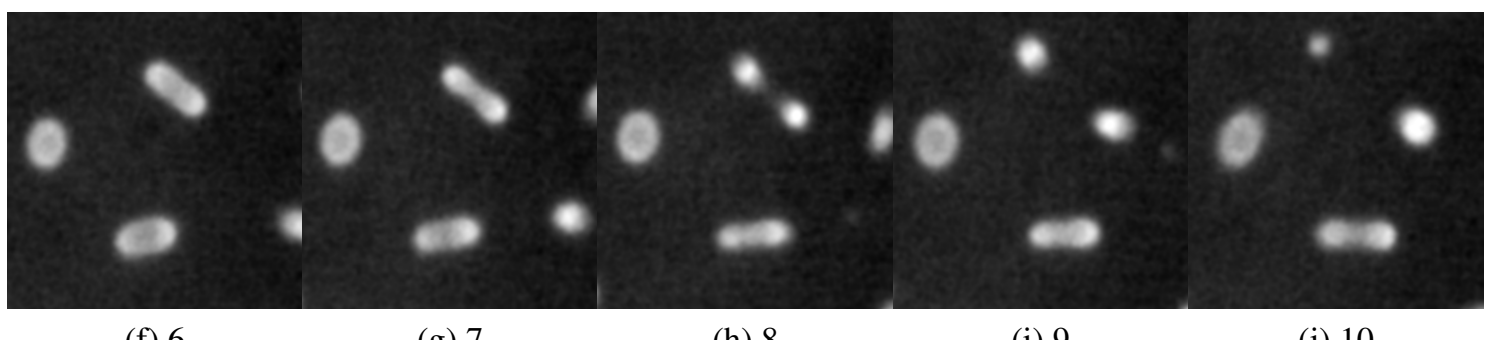

(f) 6

(g) 7

(h) 8

(i) 9

(j) 10

Figure 4: Ten consecutive snapshots for the motion of $\left(80 \% \mathrm{H}_{2}+20 \% \mathrm{CH}_{4}\right)$-air ball-like flames with a time interval, $\Delta \mathrm{t}=0.1 \mathrm{~s}$. Videos are supplied as supplementary material.

distorted cell-like flames, which do not indicate an abrupt change of combustion regimes across these boundaries because the transition between combustion regimes can not be clearly defined in the experiments. However, the boundary line between ball-like and distorted cell-like flames is very clear in the experiments. The distorted cell-like flames break into several ball-like flames at a certain equivalence ratio. The ball-like flames can only be obtained in a very narrow range of equivalence ratio. This narrow range of equivalence ratio increases with the decrease of Lewis number. The main difference found for the combustion behavior of mixtures with different $\mathrm{H}_{2}$ contents in the fuel gases is the change in size and shapes of flamelets at lean limit conditions. With increasing $\mathrm{H}_{2}$ content in the fuel gases, the lean limit flamelets become smaller and evolve from a flattened to a ball-like shape, with more uniform flame front intensity of chemiluminescence.

To stabilize the flamelets below the perforated plate, small perturbations are added to the flow field using a randomly corrugated wire mesh installed just below the perforated plate. Bottom views of stable flame patterns for mixtures with different $\mathrm{H}_{2}$ contents are presented in Fig. 6. For $\left(20 \% \mathrm{H}_{2}+80 \% \mathrm{CH}_{4}\right.$ )-air flames (Fig. 6(a)), only one flamelet is located below the middle part of the perforated plate. Several flames with irregular shape are distributed near the tube wall. The irregular shape of these flames can be attributed to the influence of the tube wall. In the mixtures with higher $\mathrm{H}_{2}$ content, several flamelets appear around the middle part of the perforated plate. 


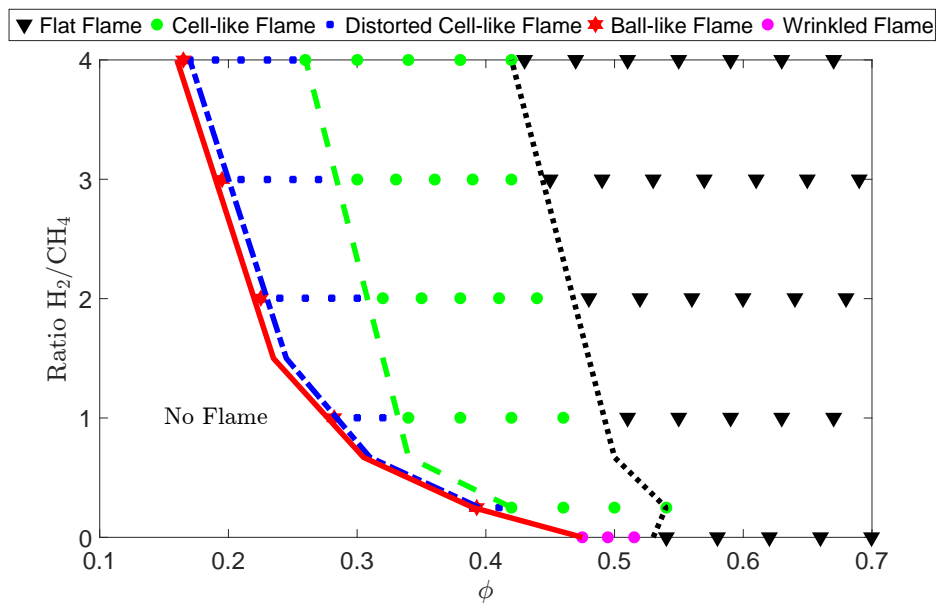

Figure 5: Experimental combustion regimes with different $\mathrm{H}_{2}$ contents in the fuel gases as a function of equivalence ratio.

The shapes of the flamelets in the middle part of the perforated plate are nearly identical, while the shapes of the flamelets near the tube wall slightly differ from those located in the middle part of the perforated plate. Furthermore, the flame patterns in the mixtures with higher $\mathrm{H}_{2}$ contents tend to form a planar, polygonal array of flames, even though the spatial flow variations introduced by the mesh are of a random character. However, some flamelets below the middle part of the perforated plate are "missing" from the overall polygonal pattern, since the stability of the flames is sensitive to flow fluctuations.

Figure 7 displays the Abel-inverted $\mathrm{CH}^{*}$ chemiluminescence images of selected flamelets stabilized around the middle part of the perforated plate in the mixtures with different $\mathrm{H}_{2}$ contents, taken from the side view. It shows that the lean limit flames with $20 \%, 40 \%$ and $60 \% \mathrm{H}_{2}$ contents have oblate spheroidal, closed shapes with a non-uniform flame front: natural chemiluminescence is stronger at more curved flame front segments. The width of the lean limit flame decreases considerably with the increase of $\mathrm{H}_{2}$ content from $20 \%$ to $40 \%$, and remains nearly the same with a further increase of $\mathrm{H}_{2}$ content from $40 \%$ to $60 \%$. It should be noted that the size (half width $=$ $2.1 \mathrm{~mm}$ ) of the ball-like flames in the $40 \% \mathrm{H}_{2}$-containing mixture is larger than that (half width $=$ $1.8 \mathrm{~mm}$ ) in a tube with an inner diameter of $13.5 \mathrm{~mm}$ [14]. As for the lean limit flame with $80 \%$ $\mathrm{H}_{2}$ content, it has a nearly spherical shape, and the intensity of the flame front becomes relatively uniform. 


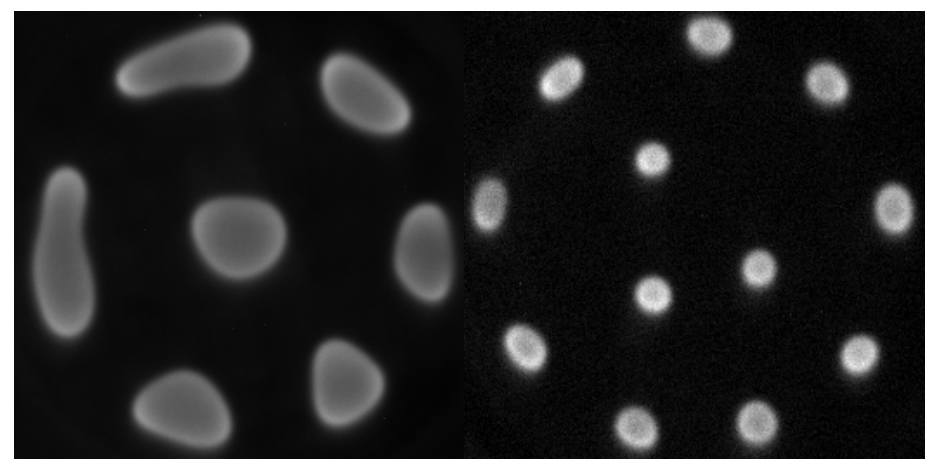

(a) $\phi=0.39,20 \% \mathrm{H}_{2}$

(b) $\phi=0.309,40 \% \mathrm{H}_{2}$

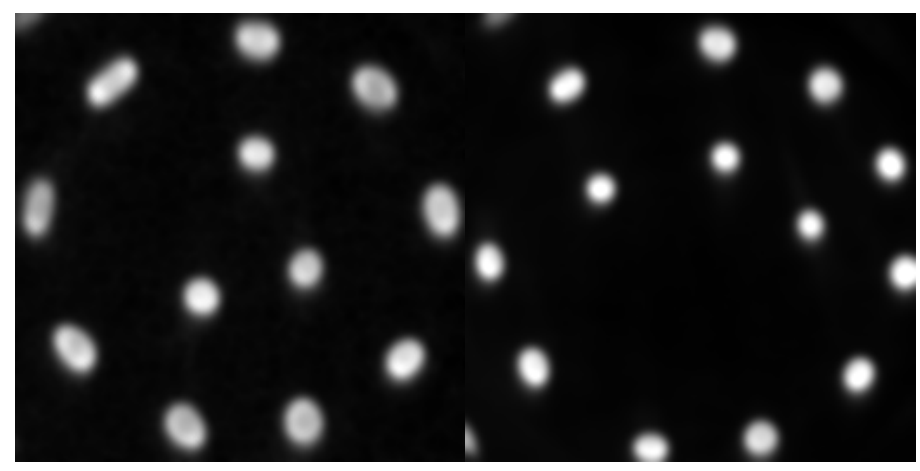

(c) $\phi=0.235,60 \% \mathrm{H}_{2}$

(d) $\phi=0.16,80 \% \mathrm{H}_{2}$

Figure 6: Flame patterns with different $\mathrm{H}_{2}$ contents in the fuel gases.

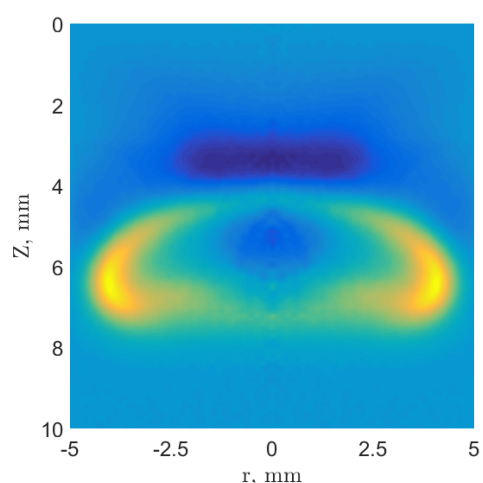

(a) $\phi=0.39$

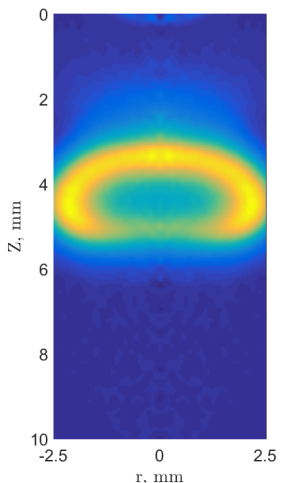

(b) $\phi=0.309$

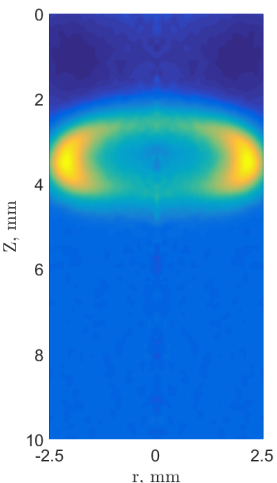

(c) $\phi=0.235$

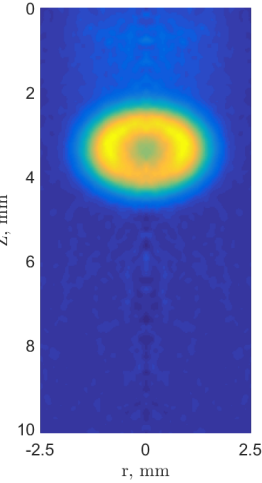

(d) $\phi=0.16$

Figure 7: The Abel-inverted $\mathrm{CH}^{*}$ chemiluminescence of the ball-like lean limit flames with different $\mathrm{H}_{2}$ contents in the fuel gases. (a): $20 \% \mathrm{H}_{2}$, (b): $40 \% \mathrm{H}_{2}$, (c): $60 \% \mathrm{H}_{2}$ and (d): $80 \% \mathrm{H}_{2}$.

To gain more insights into the characteristics of these limit flames with different $\mathrm{H}_{2}$ contents and contrast them with the ball-like flame in a narrow tube having an inner diameter of 13.5 $\mathrm{mm}$ [14], detailed numerical simulations were conducted. The numerical approach and simulation results are discussed in the following two sections. 


\section{Numerical approach}

The laminar flames under consideration are mathematically described by the conservation equations of mass, momentum, energy, and species in a cylindrical coordinate system. The governing equations are omitted here for brevity, but they are described in our previous publication [14]. The flow is treated as a mixture of perfect gases under the assumption of an ideal gas equation of state. The set of governing equations is solved by the body-fitted, multi-block, adaptive mesh refinement, finite-volume framework that has been originally developed by Groth and co-researchers $[14,19,20]$. Thermodynamic properties, transport properties and species net production/destruction rates are all computed by the open-source library CANTERA [21].

The pattern of the stable lean limit flames that form below the perforated plate closely resembles a planar, polygonal array of laminar flames, as shown in the above section. The numerical simulation of a set of lean limit flames is simplified by considering a single flame in a cylindrical domain with a diameter which equals the characteristic distance between the centers of adjacent flame elements in such array. Due to this approximation, the numerical simulations only provide qualitative understanding of the experimental results. Also, the complex dynamics (e.g., splitting and extinction) of multiple ball-like flames cannot be predicted by the present steady simulations. The computational domain is a two-dimensional cylindrical channel with a height of $3 \mathrm{~cm}$ (see Fig. 8) and the radius is determined by averaging the distance between the centers of adjacent, stable, ball-like lean limit flames recorded in the experiments and taking half of the mean distance. The determined radii are specified in Table 2. The left boundary of the domain corresponds the axis of symmetry of the flame. The right boundary is treated as a symmetry one, which can also be seen as a slip and adiabatic wall (zero-gradient conditions for temperature and species mass fractions). The upper boundary is assumed to be iso-thermal with a temperature of $300 \mathrm{~K}$ and accounts for the diffusion of fuel/air mixture across the boundary, whereas the lower boundary is modelled as a free outflow.

The diffusion velocity is computed using the mixture-averaged transport model. The skeletal mechanism for $\mathrm{CH}_{4}$ of Smooke et al. [22] is employed in the present simulations, which consists of 16 species and 25 elementary reactions. These transport and chemical models obtained good 
qualitative results in the simulation of ball-like lean limit flames in our previous research [13, 14]. Gravity $\left(\mathrm{g}=9.81 \mathrm{~m} / \mathrm{s}^{2}\right)$ and radiative heat loss are taken into consideration. Thermal radiation is modelled via the optically-thin Planck model. The Planck mean absorption coefficient of the mixture is evaluated from the major radiating species $\mathrm{CO}_{2}, \mathrm{H}_{2} \mathrm{O}$ and $\mathrm{CO}$. The individual coefficients are calculated using a statistical narrow-band model and the dataset of Soufiani and Taine [23].

The conditions for the simulated cases are summarized in Table 2. Note that in order to assess the tube diameter and boundary condition effects on the prediction of the lean limit flames, two extra cases $(\mathrm{D}, \mathrm{E})$ for the lean limit flames inside a $13.5 \mathrm{~mm}$ diameter tube with different right boundary conditions are included. The numerical solutions are obtained with up to three levels of mesh refinement, achieving a minimum cell size of $54 \mu \mathrm{m}$ for all the cases. These levels of resolution are found to be sufficient to get grid-independent solutions. Local time-stepping along with the low-Mach number preconditioner described by Weiss and Smith [24] and the explicit multi-stage optimally-smoothing time marching scheme of van Leer et al. [25] were used to obtain the time-invariant solutions. For each case, a solution (flat flame) was first obtained at a higher equivalence ratio and this solution was then utilized as the initial condition for a new computation with a slight decrease in equivalence ratio. The procedure was repeated until a solution (balllike flame) for the limit equivalence ratio was achieved. In each computation, the $\mathrm{L}_{2}$-norm of the residuals for density and momentum was driven below $1 \times 10^{-6}$.

Table 2: Summary of conditions for the simulated cases. $R_{0}$ is the radius of the domain.

\begin{tabular}{cccccc}
\hline Case & Fuel & $V_{\text {in }}(\mathrm{cm} / \mathrm{s})$ & $\phi$ & $R_{0}(\mathrm{~mm})$ & Right Boundary Condition \\
\hline $\mathrm{A}$ & $40 \% \mathrm{H}_{2}+60 \% \mathrm{CH}_{4}$ & 0.72 & 0.316 & 8 & Symmetry \\
B & $60 \% \mathrm{H}_{2}+40 \% \mathrm{CH}_{4}$ & 0.73 & 0.25 & 7.5 & Symmetry \\
$\mathrm{C}$ & $80 \% \mathrm{H}_{2}+20 \% \mathrm{CH}_{4}$ & 0.73 & 0.18 & 7 & Symmetry \\
$\mathrm{D}$ & $40 \% \mathrm{H}_{2}+60 \% \mathrm{CH}_{4}$ & 0.72 & 0.315 & 6.75 & Symmetry \\
E & $40 \% \mathrm{H}_{2}+60 \% \mathrm{CH}_{4}$ & 0.72 & 0.315 & 6.75 & No-slip, iso-thermal wall \\
\hline
\end{tabular}

\section{Numerical lean limit flames and discussion}

The experimentally-observed, stable, ball-like, lean limit flames that formed below the perforated plate are modelled by simulating single lean limit flames in domains with an adiabatic slip 


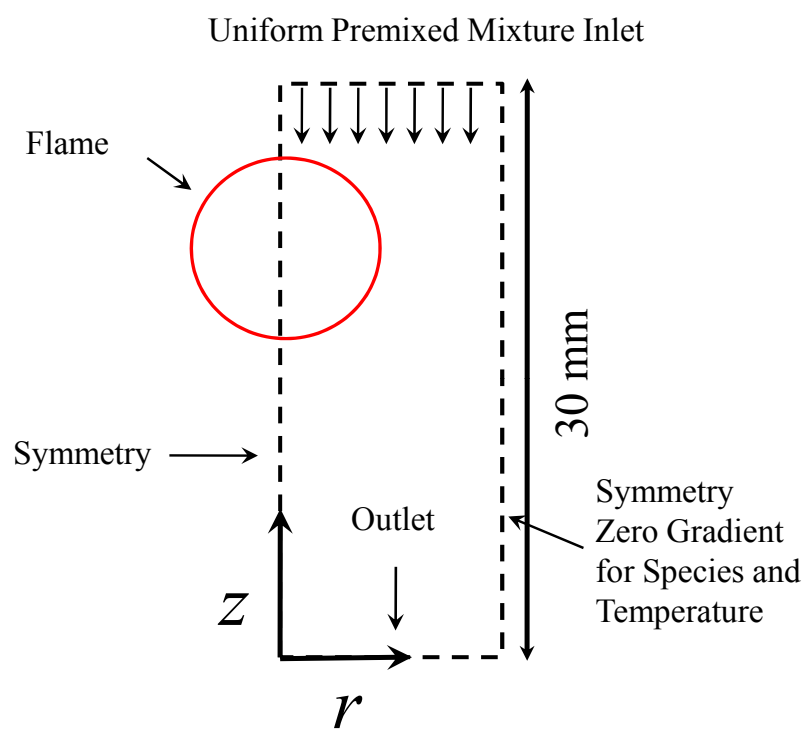

Figure 8: Schematic of the computational domain and boundary conditions.

wall (symmetry) boundary to represent the symmetry between adjacent ball-like fronts. The radii of the domains for the $40 \%, 60 \%$ and $80 \% \mathrm{H}_{2}$-content in the fuel are $8 \mathrm{~mm}, 7.5 \mathrm{~mm}$ and $7 \mathrm{~mm}$, respectively. In addition, for comparison, two ball-like lean flames (Cases D and E) in a tube with an inner diameter of $13.5 \mathrm{~mm}$ are simulated with adiabatic slip wall (symmetry) and no-slip iso-thermal wall boundary conditions, respectively. Figure 9 displays the distributions of chemical heat release rate and streamlines for the computed ball-like lean limit flames at the various conditions. It is seen that the predicted lean limit flames (a-d) in the domains with the adiabatic slip wall have ellipsoidal shapes, which are qualitatively similar to the lean limit flames recorded in the experiments. However, the predicted lean limit flame (e) in the domain with the no-slip iso-thermal wall displays a shape closer to a spherical one. Figure 9 also shows that, by adding more $\mathrm{H}_{2}$ to the fuel, the size of the lean limit flame decreases, which is consistent with the experimental observations (Fig. 7). For all the predicted lean limit flames, the flame size and the lean flammability limit are, nevertheless, slightly larger than those measured in the experiments. Also, the experimental lean limit flamelet in the mixture with $80 \% \mathrm{H}_{2}$ is more spherical and has a more uniform front than the simulated one. These discrepancies could be attributed to uncertainties in the chemical kinetic and transport models, as the prediction of the lean limit flames is very sensitive to the transport 
and chemistry models [14]. Besides, the simplified approach to represent the interaction between adjacent flame elements in the array of ball-like flames is just an approximation.

Figure 9 further shows that all the simulated lean limit flames are located inside a recirculation zone which is induced by the buoyancy force acting on the hot gases as the flame burning velocity becomes small. This result suggests that such a recirculation zone is also an attribute of lean limit flamelets of $\mathrm{H}_{2}-\mathrm{CH}_{4}$-air mixtures stabilized below the perforated plate in the experiments. The recirculation zone is probably a key factor which makes the formation of the ball-like flames at normal gravity possible. If the recirculation is not too strong, it forms a region with low speed, which minimizes the role of convection. However, if the recirculation velocity is strong, it may distort ball-like flames making them non-spherical and non-uniform. As shown in Table 3, the maximum upward velocity ( $\left.V_{\text {up }}\right)$ that is induced by buoyancy along the centerline is significantly larger for the flames that are part of the array, compared to that of the single flames in the tube with a diameter of $13.5 \mathrm{~mm}$. Therefore, the ellipsoidal shape of the lean limit flames attained in the present experiments using a larger tube can be explained by the stronger impact of buoyancy, as compared to its counterpart in the narrow tube. Besides, for the predicted lean limit flames that are part of the array, the heat released from the flame front is almost completely removed by heat convection through the tube outlet. The integrated heat loss by radiation is less than $3.5 \%$ of the integrated chemical heat release, as shown in Table 4. This indicates that the stability of the multiple ball-like lean limit flamelets at normal-gravity conditions is determined by convective heat loss. This differs from micro-gravity flame balls which are stabilized by radiative heat loss.

Table 3: Maximum upward velocity $\left(V_{\text {up }}\right)$ induced by buoyancy along the centerline for the simulated cases.

\begin{tabular}{ccccc}
\hline Case & Fuel & $\phi$ & Right Boundary Condition & $V_{\text {up }} \mathrm{cm} / \mathrm{s}$ \\
\hline A & $40 \% \mathrm{H}_{2}+60 \% \mathrm{CH}_{4}$ & 0.316 & Symmetry & 9.30 \\
B & $60 \% \mathrm{H}_{2}+40 \% \mathrm{CH}_{4}$ & 0.25 & Symmetry & 8.95 \\
C & $80 \% \mathrm{H}_{2}+20 \% \mathrm{CH}_{4}$ & 0.18 & Symmetry & 8.95 \\
D & $40 \% \mathrm{H}_{2}+60 \% \mathrm{CH}_{4}$ & 0.315 & Symmetry & 9.14 \\
E & $40 \% \mathrm{H}_{2}+60 \% \mathrm{CH}_{4}$ & 0.315 & No-slip, iso-thermal wall & 6.00 \\
\hline
\end{tabular}

Figure 10 presents the variations of velocity, chemical heat release, mole fraction of $\mathrm{H}_{2}$ and $\mathrm{CH}_{4}$, and flame temperature along the centerline for the lean limit flame with $40 \% \mathrm{H}_{2}$ content 


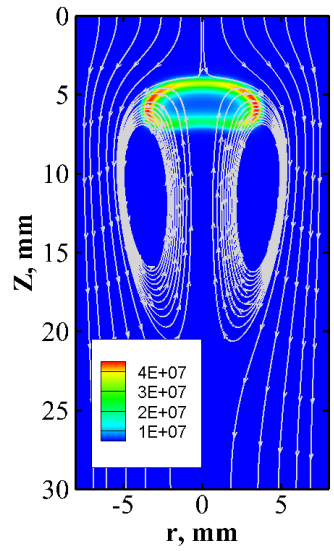

(a) Case A

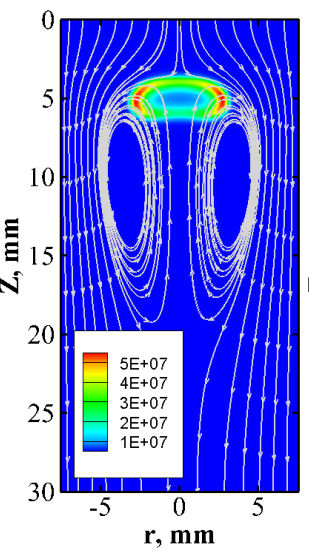

(b) Case B

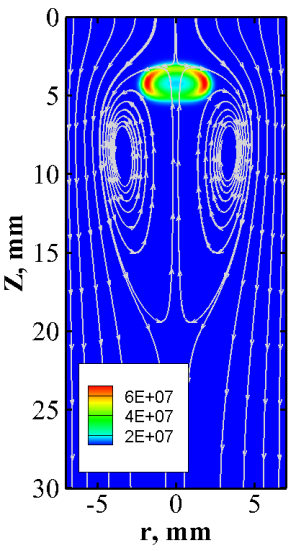

(c) Case C

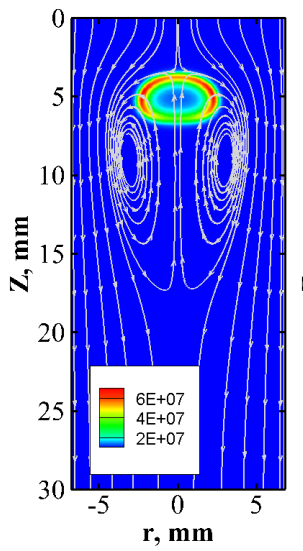

(d) Case D

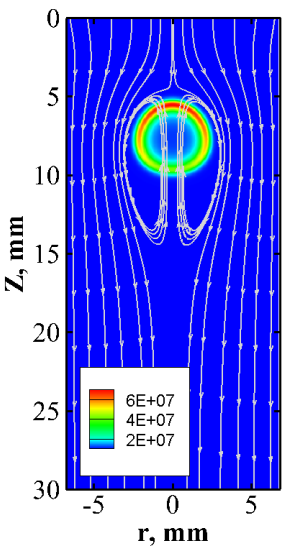

(e) Case E

Figure 9: Distribution of chemical heat release rate $\left(\mathrm{W} / \mathrm{m}^{3}\right)$ and flow streamlines for the lean limit flames in the mixtures with different $\mathrm{H}_{2}$ contents and with different domain radii and right boundary conditions.

Table 4: Integrated chemical heat release $\left(\mathrm{Q}_{\mathrm{chem}}\right)$, conductive heat loss to the tube wall $\left(\mathrm{Q}_{\text {cond }}\right)$ and radiative heat loss $\left(\mathrm{Q}_{\mathrm{rad}}\right)$ for the simulated cases.

\begin{tabular}{ccccc}
\hline Case & $\phi$ & $\mathrm{Q}_{\text {chem }}(\mathrm{W})$ & $\mathrm{Q}_{\text {cond }}(\mathrm{W})$ & $\mathrm{Q}_{\text {rad }}(\mathrm{W})$ \\
\hline $\mathrm{A}$ & 0.316 & 3.40 & 0 & 0.12 \\
$\mathrm{~B}$ & 0.25 & 2.40 & 0 & 0.061 \\
$\mathrm{C}$ & 0.18 & 1.43 & 0 & 0.027 \\
$\mathrm{D}$ & 0.315 & 2.07 & 0 & 0.060 \\
$\mathrm{E}$ & 0.315 & 2.36 & 1.47 & 0.058 \\
\hline
\end{tabular}

(Case A). It can be seen in Fig. 10(a) that the axial component of velocity displays positive values due to the occurrence of the recirculation zone. The leading edge indicated by chemical heat release locates in the low upward velocity region, while the trailing edge locates in the high upward velocity region. Figure 10(b) illustrates that a part of the unburned mixture leaks from the upstream side to the downstream side. The unburned reactants at the downstream side diffuse from the surrounding flow into the recirculation zone and are then supplied back to the flame region by the upward convective flow. Although the adiabatic laminar flame speed for a 1D flame in the corresponding lean limit mixture is small, the combustion of the ball-like lean limit flame is enhanced by strong preferential diffusion allowing the ball-like flame to burn steadily in a relatively strong flow. Besides, the burning of the trailing edge is enhanced by preheating: a large amount of the heat generated in the leading edge is transported to the trailing edge by conduction/convection in the recirculation zone. 


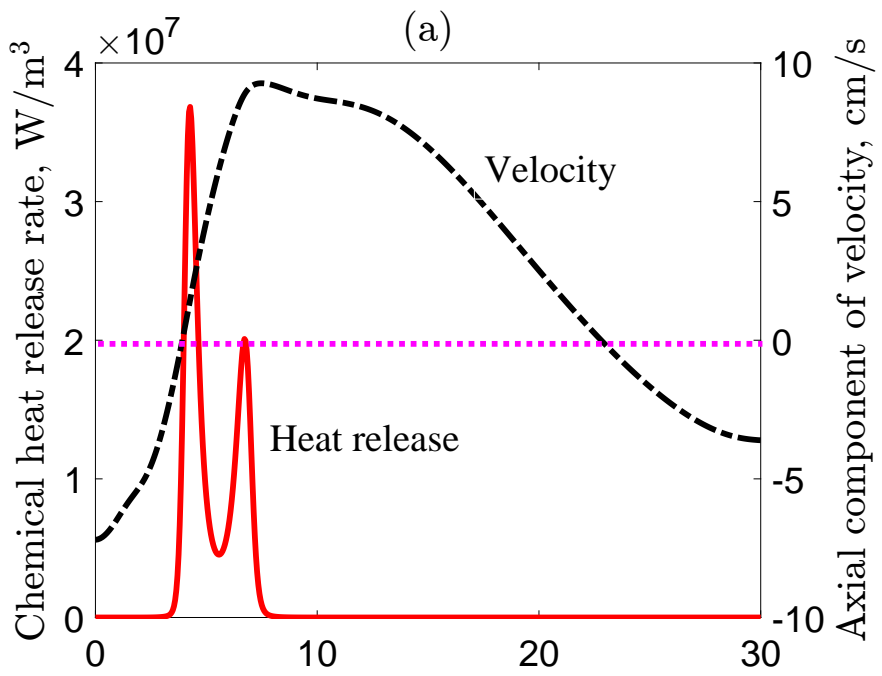

(b)

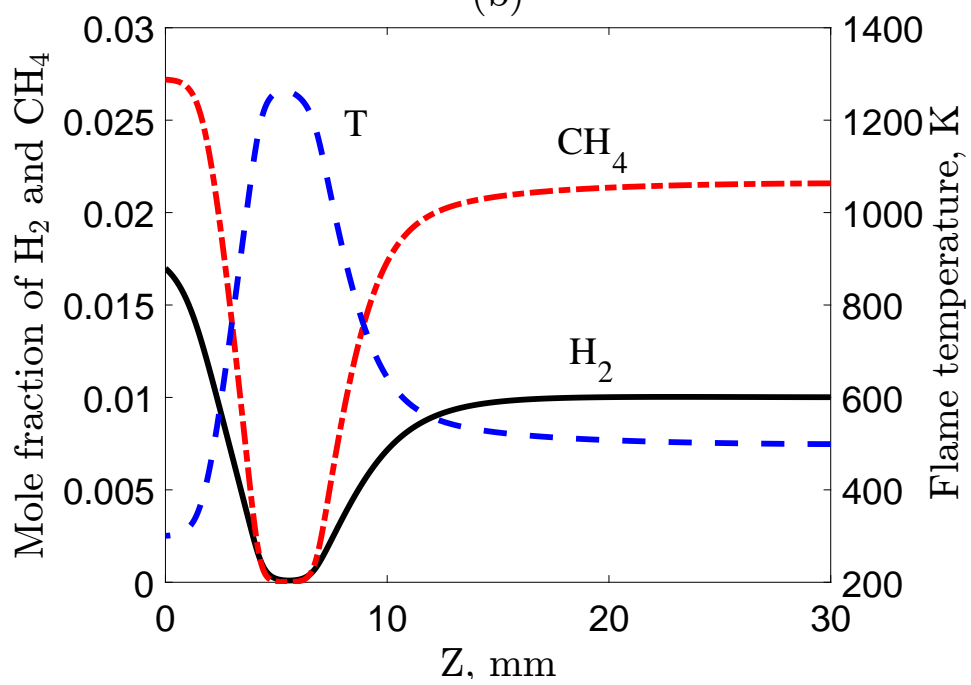

Figure 10: Distributions of velocity, chemical heat release, mole fraction of $\mathrm{H}_{2}$ and $\mathrm{CH}_{4}$ and flame temperature along the centerline for the lean limit flame with $40 \% \mathrm{H}_{2}$ content (Case A).

\section{Conclusions}

Near-limit behavior of low-Lewis-number flames burning below a perforated plate in a downflowing mixture is experimentally and numerically studied. The combustion regimes for the flames with different $\mathrm{H}_{2}$ contents in the fuel gas as a function of equivalence ratio are experimentally identified. Planar flame, cell-like flames, distorted cap-like flames, and chaotically moving ball-like flames are observed in the experiments with decreasing equivalence ratio for fuel mixtures with more than $20 \% \mathrm{H}_{2}$. To our knowledge, this is the first observation of multiple, stable, closed, ball- 
like lean limit flames at normal gravity conditions. It is found that the ball-like lean limit flames can be stabilized by adding small perturbations into the flow field using a randomly corrugated wire mesh. The $\mathrm{CH}^{*}$ chemiluminescence distributions of the stable flamelets are recorded in the experiments. These measurements reveal that, at lean flammability limits, the flamelets have an oblate spheroidal shape with a non-uniform flame front: chemiluminescence is stronger at more curved flame front segments. As the $\mathrm{H}_{2}$ content in the fuel gas is increased, the size of the flamelets decreases. At $80 \%$ of $\mathrm{H}_{2}$ in the fuel gas, the near-limit flamelets become nearly spherical, and the luminosity of the flame front becomes more uniform.

The combustion characteristics of the multiple ball-like lean limit flamelets in a downward mixture flow are further investigated by simulating single flamelets representative of the array of multiple ones at normal gravity conditions. One case corresponding to a narrow tube, with a noslip and iso-thermal wall, is also simulated for comparison. It is found that the stronger deviation of the shape of the multiple lean limit flames from the spherical one, compared to the case of a single limit flame in a narrow tube, is caused by a stronger buoyancy effect in the former case. The lean limit flamelets are always found inside a recirculation zone, which could be a key factor that allows formation of ball-like flames at normal gravity. The multiple ball-like lean limit flamelets at normal-gravity conditions are stabilized by convective heat loss, while ideal flame balls are stabilized by radiative heat loss. Overall, the wall confinement is not crucial for the formation of ball-like flames.

\section{Acknowledgments}

The financial support of the Dutch Technology Foundation (STW), Project 13549, is gratefully acknowledged. The authors thank Prof. Clinton Groth for providing access to the CFFC (Computational Framework for Fluids and Combustion) code.

\section{References}

[1] A. L. Sánchez, F. A. Williams, Recent advances in understanding of flammability characteristics of hydrogen, Prog. Energy Combust. Sci. 41 (2014) 1-55. 
[2] Y. Zeldovich, Theory of combustion and detonation of gases, Academy of Sciences (USSR), 1944.

[3] P. D. Ronney, Near-limit flame structures at low Lewis number, Combust. Flame 82 (1) (1990) 1-14.

[4] P. D. Ronney, K. N. Whaling, A. Abbud-Madrid, J. L. Gatto, V. L. Pisowicz, Stationary premixed flames in spherical and cylindrical geometries, AIAA J. 32 (3) (1994) 569-577.

[5] M. Abid, M. Wu, J. Liu, P. Ronney, M. Ueki, K. Maruta, H. Kobayashi, T. Niioka, D. Vanzandt, Experimental and numerical study of flame ball IR and UV emissions, Combust. Flame 116 (3) (1999) 348-359.

[6] P. D. Ronney, M. S. Wu, H. G. Pearlman, K. J. Weiland, Experimental study of flame ball in space: preliminary results from STS-83, AIAA J. 36 (8) (1998) 1361-1368.

[7] L. Kagan, G. Sivashinsky, Self-fragmentation of nonadiabatic cellular flames, Combust. Flame 108 (1-2) (1997) 220-226.

[8] I. Brailovsky, G. Sivashinsky, On stationary and travelling flame balls, Combust. Flame 110 (4) (1997) 524-529.

[9] R. Fursenko, S. Minaev, Flame balls dynamics in divergent channel, Combust. Theor. Model. 15 (6) (2011) 817-825.

[10] K. Takase, X. Li, H. Nakamura, T. Tezuka, S. Hasegawa, M. Katsuta, M. Kikuchi, K. Maruta, Extinction characteristics of $\mathrm{CH} 4 / \mathrm{O} 2 / \mathrm{Xe}$ radiative counterflow planar premixed flames and their transition to ball-like flames, Combust. Flame 160 (7) (2013) 1235-1241.

[11] R. Fursenko, S. Minaev, H. Nakamura, T. Tezuka, S. Hasegawa, T. Kobayashi, K. Takase, M. Katsuta, M. Kikuchi, K. Maruta, Near-lean limit combustion regimes of low-Lewisnumber stretched premixed flames, Combust. Flame 162 (5) (2015) 1712-1716.

[12] Y. L. Shoshin, L. P. H. de Goey, Experimental study of lean flammability limits of methane/hydrogen/air mixtures in tubes of different diameters, Exp. Therm Fluid Sci. 34 (3) (2010) 373-380.

[13] Y. Shoshin, J. van Oijen, A. Sepman, L. P. H. de Goey, Experimental and computational study of the transition to the flame ball regime at normal gravity, Proc. Combust. Inst. 33 (1) (2011) 1211-1218. 
[14] F. E. Hernández-Pérez, B. Oostenrijk, Y. Shoshin, J. A. van Oijen, L. P. H. de Goey, Formation, prediction and analysis of stationary and stable ball-like flames at ultra-lean and normal-gravity conditions, Combust. Flame 162 (4) (2015) 932-943.

[15] G. E. Markstein, Nonsteady flame propagation, Pergamon Press, Oxford, 1964.

[16] B. Bregeon, A. S. Gordon, F. A. Williams, Near-limit downward propagation of hydrogen and methane flames in oxygennitrogen mixtures, Combust. Flame 33 (C) (1978) 33-45.

[17] H. F. Coward, F. Brinsley, The dilution-limits of inflammability of gaseous mixtures, J. Chem. Soc., Trans. 105 (0) (1914) 1859-1885.

[18] S. F. Wang, H. Zhang, J. Jarosinski, A. Gorczakowski, J. Podfilipski, Laminar burning velocities and Markstein lengths of premixed methane/air flames near the lean flammability limit in microgravity, Combust. Flame 157 (4) (2010) 667-675.

[19] X. Gao, C. P. Groth, A parallel solutionadaptive method for three-dimensional turbulent nonpremixed combusting flows, J. Comput. Phys. 229 (9) (2010) 3250-3275.

[20] F. Hernández-Pérez, F. Yuen, C. Groth, Ö. Gülder, LES of a laboratory-scale turbulent premixed Bunsen flame using FSD, PCM-FPI and thickened flame models, Proc. Combust. Inst. 33 (1) (2011) 1365-1371.

[21] California Institute of Technology, CANTERA Release 1.7 (2006).

[22] Reduced kinetic mechanisms and asymptotic approximations for methane/air flames, in: M. D. Smooke (Ed.), Lecture Notes in Physics, Springer-Verlag Heidelberg, 1991, pp. 120.

[23] T. J. Soufiani A., High temperature gas radiative property parameters of statistical narrowband model for $\mathrm{H} 2 \mathrm{O}, \mathrm{CO} 2$ and $\mathrm{CO}$, and correlated-K model for $\mathrm{H} 2 \mathrm{O}$ and $\mathrm{CO} 2$, Int. J. Heat Mass Transfer 40 (4) (1997) 987-991.

[24] J. M. Weiss, W. A. Smith, Preconditioning applied to variable and constant density flows, AIAA J. 33 (11) (1995) 2050-2057.

[25] B. van Leer, C. H. Tai, K. G. Powell, Design of optimally-smoothing multi-stage schemes for the Euler equations, Paper 89-1933-CP, AIAA (June 1989). 